\title{
Collision Avoidance Maneuver Planning Using GA for LEO and GEO Satellite Maintained in Keeping Area
}

\section{Sang-Cherl Lee* and Hae-Dong Kim*}

Aerospace Convergence Technology Research Laboratory, Korea Aerospace Research Institute, Daejeon, 305-806, Korea,

\author{
Jinyoung Suk** \\ Department of Aerospace Engineering, Chungnam National University, Daejeon, 305-764, Korea,
}

\begin{abstract}
In this paper, a collision avoidance maneuver was sought for low Earth orbit (LEO) and geostationary Earth orbit (GEO) satellites maintained in a keeping area. A genetic algorithm was used to obtain both the maneuver start time and the delta- $\mathrm{V}$ to reduce the probability of collision with uncontrolled space objects or debris. Numerical simulations demonstrated the feasibility of the proposed algorithm for both LEO satellites and GEO satellites.
\end{abstract}

Key words: Geostationary Satellite, Low Earth Orbit Satellite, Collision Avoidance, Genetic Algorithm, Space Debris, Avoidance Maneuver.

\section{Introduction}

Since the launch created the risk of collision between the debris and the operational satellites. According to NASA Orbital Debris Quarterly News, these two breakups created approximately 5000 objects larger than $10 \mathrm{~cm}$ in diameter and increased the debris cataloged by $50 \%[1,2,3]$.

The Korea Aerospace Research Institute (KARI) operates KOMPSAT 2 and KOMPSAT 3 as low Earth orbit (LEO) satellites and COMS in geostationary Earth orbit (GEO). KARI also has plans to launch KOMPSAT 5 and KOMPSAT 3A in LEO and GEOKOMPSAT 2A and GEOKOMPSAT 2B in GEO in the future. Collision risks have been increasing not only in LEO but also in GEO. For example, KOMPSAT 2 received 16 Conjunction Summery Messages (CSM) from JSpOC in 2011, but because of the non-acquisition of precise tracking data for the approaching objects and the lack of a collision avoidance maneuver planning system, no action was taken. However, the COMS (Chollian) completed a maneuver to avoid collision and maintain a safe distance from the approaching Russian satellite RADUGA 1-7 when the latter operated abnormally in February 2011, although this maneuver was done without considering collision probability, position uncertainty, and precise tracking data for RADUGA 1-7.

As the amount of space debris increases, collision avoidance maneuvers are required for operational satellites. The lower the probability threshold, the more maneuvers shall be performed. However, no matter how many maneuvers are performed, risk can never be completely eliminated. Therefore, we can acquire avoidance maneuvers by considering the multi-objective function to decrease collision probability and decrease maneuver magnitude.

Several studies have been undertaken in Korea. For example, an optimal collision avoidance maneuver was investigated that considered multi-threatening objects within short periods while satisfying fuel constraints which provided an acceptable collision probability for LEO satellites[4, 5, 6]. Analysis has also been performed on the collision probability of the COMS-1 satellite according to position uncertainty, and analysis has been performed on the collision avoidance maneuver frequency for KOMPSAT-2 and KOMPSAT-5[7, 8]. Meanwhile, owing to the growing risk of space collision,
This is an Open Access article distributed under the terms of the Creative Commons Attribution Non-Commercial License (http://creativecommons.org/licenses/by$\mathrm{nc} / 3.0 /$ which permits unrestricted non-commercial use, distribution, and reproduction in any medium, provided the original work is properly cited.

\footnotetext{
(c) * Senior Researcher

** Associate Professor, Department of Aerospace Engineering Corresponding author : jsuk@cnu.ac.kr
} 
analysis tools to mitigate the risk of space conjunction for our satellites have been developed by KARI. The conceptual design of space debris collision risk management software has been presented[9].

The solution method for the collision avoidance maneuver can be done with various optimization algorithms. It has been performed using derivative-based methods. The disadvantage of the above optimization techniques is that they are based on an initial guess and may be trapped in the local minimum. Recently, the research community has diverted their attention towards soft computing techniques, such as evolutionary algorithms and genetic algorithms. Genetic algorithms are search algorithms based on the mechanism of natural selection and natural genetics. GA modeling is gaining importance because of its robust random search capability and near global optimal values. For example, in a recent study by M. Duncan and J. Wysackin regards to finding an optimal avoidance maneuver that mitigates the risk of collision events, a derivative-free optimization (DFO) technique was utilized[10]. DFO is an unconstrained multi-variable optimization technique. It iteratively forms a quadratic model that produces a local approximation of the cost function. The constraints are simply added to the cost function to apply an additional cost or "penalty" as required. However, a genetic algorithm is a constrained multi-variable optimization technique. Genetic algorithms are used to acquire both the maneuver start time and the delta- $\mathrm{V}$ to decrease the collision probability from an approaching uncontrolled object with consideration of orbit determination accuracy or position uncertainty. Characteristics of genetic algorithms include stochastic iteration, parallel processing, basis on population, absence of gradients, integer constraints, and an automatic or usersupplied start population[11].

The rest of this paper is organized as follows. Section 2 details stationkeeping for GEO satellites and ground track maintenance for LEO satellites, the use of genetic algorithms, and planning for collision avoidance maneuvers. Section 3 presents the results of a numerical simulation based on the maneuver strategy employed. Finally, conclusions are described.

\section{Avoidance Maneuvers Planning for LEO/ GEO Satellites}

\subsection{GEOStationkeeping and LEO Ground Track Maintenance}

The ground track of a LEO satellite will drift by longitude at our desired crossing point over time owing to external perturbation. Due to the gravitational force, solar wind, and atmospheric drag, deviation from the reference orbit leads to ground track drift. Ground track error is the amount of drift of the current orbit from the reference orbit crossing the equator westward or eastward. Therefore, a ground track maintenance maneuver should be undertaken to maintain the ground track within a predefined control band around the reference ground track. For example, in-plane maneuvers are used for altitude adjustment to compensate for the effect of air-drag. However, this decrease in altitude affects ground track repeatability or ground track error.

The design objective was to choose the semi-major axis so that the desired $\omega_{\mathrm{S}} / \omega_{\mathrm{E}}^{*}$ ratio for a repeating ground track is achieved. Rather than solving for one value of the semimajor axis, a range of possible values is considered around the nominal semi-major axis[12]. The mean semi-major axis, mean eccentricity, mean orbital inclination and mean argument of perigee which satisfies the user's repeating ground track criteria can be solved by Equations (1) -(4).

$$
\frac{\omega_{\mathrm{s}}}{\omega_{\mathrm{E}}^{*}}=\frac{\sqrt{\frac{\mu}{\mathrm{a}^{3}}+\dot{\omega}+\dot{M}}}{\omega_{\mathrm{E}}-\dot{\Omega}}
$$

Here, $\omega_{\mathrm{s}}$ denotes the mean orbital rate of the satellite, and $\omega_{\mathrm{E}}^{*}=\omega_{\mathrm{E}}-\dot{\Omega}$ indicates the rotation rate of Earth with respect to the orbital plane; $\dot{\omega}, \dot{M}$ and $\dot{\Omega}$ are expressed as

$$
\begin{aligned}
& \dot{\omega}=\frac{\dot{-3 n J_{2} \mathrm{R}_{\mathrm{e}}^{2}}}{2 \mathrm{a}^{2}\left(1-\mathrm{e}^{2}\right)^{2}}\left(2.5 \sin ^{2} \mathrm{i}-2\right) \\
& \dot{\mathrm{M}}=\frac{\dot{-3 n J_{2} \mathrm{R}_{\mathrm{e}}^{2}}}{2 \mathrm{a}^{2}\left(1-\mathrm{e}^{2}\right)^{2}}\left(1.5 \sin ^{2} \mathrm{i}-1\right) \sqrt{1-\mathrm{e}^{2}} \\
& \dot{\Omega}=\frac{-3 \mathrm{~nJ}_{2} \mathrm{R}_{\mathrm{e}}^{2}}{2 \mathrm{a}^{2}\left(1-\mathrm{e}^{2}\right)^{2}} \cos \mathrm{i}
\end{aligned}
$$

where a, e, $\mathrm{i}, \mathrm{n}, \mathrm{R}_{\mathrm{e}}, \mu$, and $\mathrm{J}_{2}$ are the semi-major axis, eccentricity, inclination, mean orbital rate, Earth's radius, gravitational constant, and the perturbation from Earth's gravity, respectively; $\omega_{\mathrm{E}}, \dot{\omega}, \dot{\mathrm{M}}$, and $\dot{\Omega}$ denote the mean rotation rate of Earth, the time derivative of the argument of perigee, the time derivative of the mean anomaly, and the time derivative of right ascension of an ascending node, respectively.

The repeating ground track error is the difference between the current longitude and the nearest longitude grid point computed as a point of an orbit with a specified repeat count and reference longitude. The repeat count gives the number of orbits before the ground track repeats over the same longitude. Therefore, the amount of delta- $\mathrm{V}$ required 
is considered to maintain the repeating ground track when a satellite needs to avoid other objects while remaining within the ground track boundary as shown in Fig. 1. Moreover, a sun-synchronous LEO satellite should be maintained within the designated ground track boundary.

In the case of a GEO satellite, the maneuver is performed as a consequence of thruster burns orthogonal to the orbital plane that must be executed to compensate for the effect of the lunar/solar gravitation that perturbs the orbit pole by typically $0.85^{\circ}$ per year. This part of GEO stationkeeping is called North-South stationkeeping. On the other hand, EastWest stationkeeping is the management of the orbital period and eccentricity vector, and is performed by making thruster burning tangential to the orbit. Calculation of delta- $\mathrm{V}$ should also be considered, in order to simultaneously keep the orbital period synchronous with Earth's rotation and maintain the desired eccentricity. The eccentricity (i.e., the eccentricity vector) is perturbed by solar radiation pressure. A GEO satellite is stationed at $128.2^{\circ} \mathrm{E}$ within a $\pm 0.05^{\circ}$ stationkeeping box as shown in Fig. 2. However, because of the nonspherical nature of the geopotential field, the GEO satellite is attracted to a stable point that is located at approximately $75.2^{\circ} \mathrm{E}$. Therefore, East-West stationkeeping should be taken into account to control eccentricity due to solar radiation pressure and longitudinal drift as a result of the nonspherical nature of the geopotential field. The linearized equations can be obtained in terms of the eccentricity vector, inclination vector, and longitude variation with each velocity component. The required velocity increment fluctuates between 40 and $51 \mathrm{~m} / \mathrm{s}$ per year for inclination stationkeeping and up to $2 \mathrm{~m} / \mathrm{s}$ per year for longitude stationkeeping[13, 14, 15]. The maneuver time and delta-V can be acquired to keep their position maintained in stationkeeping box by solving the system of nonlinear equations defined by Equations (5) $-(7)$.

$$
\begin{aligned}
& \Delta \mathrm{V}_{\mathrm{R}}=\mathrm{V}_{\text {syn }}\left(\Delta \mathrm{e}_{\mathrm{x}} \sin \alpha-\Delta \mathrm{e}_{\mathrm{y}} \cos \alpha\right) \\
& \Delta \mathrm{V}_{\mathrm{T}}=\mathrm{V}_{\text {syn }}\left(\Delta \lambda+2 \Delta \mathrm{e}_{\mathrm{x}} \cos \alpha+2 \Delta \mathrm{e}_{\mathrm{y}} \sin \alpha\right) \\
& \Delta \mathrm{V}_{\mathrm{N}}=\mathrm{V}_{\text {syn }}\left(\Delta \mathrm{i}_{\mathrm{x}} \cos \alpha+\Delta \mathrm{i}_{\mathrm{y}} \sin \alpha\right)
\end{aligned}
$$

Here, $\Delta V_{R}, \Delta V_{T}$, and $\Delta V_{N}$ are the velocity increment in radial, tangential, and normal directions in orbit, respectively; $\alpha, \mathrm{V}_{\text {syn }}$, and $\Delta \lambda$ are are the right ascension, the nominal velocity in GEO, and the longitude difference, respectively; $\Delta \mathrm{e}_{\mathrm{x}}, \Delta \mathrm{e}_{\mathrm{y}}, \Delta \mathrm{i}_{\mathrm{x}}$, and $\Delta \mathrm{i}_{\mathrm{y}}$, and are the eccentricity and inclination vectors in the Earth Centered Inertial (ECI) coordinate system $\Delta \mathrm{e}_{\mathrm{x}}, \Delta \mathrm{e}_{\mathrm{y}}, \Delta \mathrm{i}_{\mathrm{x}}, \Delta \mathrm{i}_{\mathrm{y}}$ and $\Delta \lambda$ can be calculated with linearization.

\subsection{Genetic Algorithms}

Genetic algorithms are a global search method for solving optimization problems and were introduced in 1975 by John Holland. Genetic algorithms work for continuous, discrete, and combinatorial problems. Also, they are less susceptible to convergence at a local minimum or maximum than gradient search methods. However, they have a disadvantage in terms of their large computation time. Nevertheless, genetic algorithms offer the benefit of finding a global solution in a constrained nonlinear system. A genetic algorithm creates a population of solutions and applies genetic operators such as mutation and crossover to evolve solutions in order to find the best one. The three most important aspects of using genetic algorithms are the definition of an objective function, definition and implementation of a genetic representation, and definition and implementation of genetic operators. Genetic algorithms are iterative schemes where the population is modified using the best features

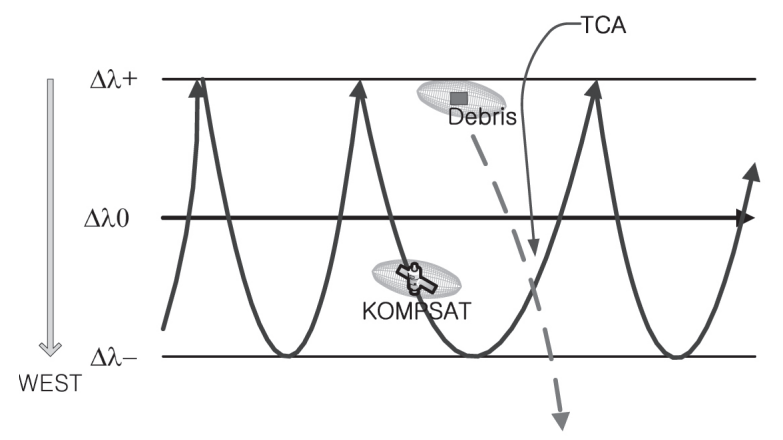

Fig. 1. Ground Track Keeping for LEO Satellites

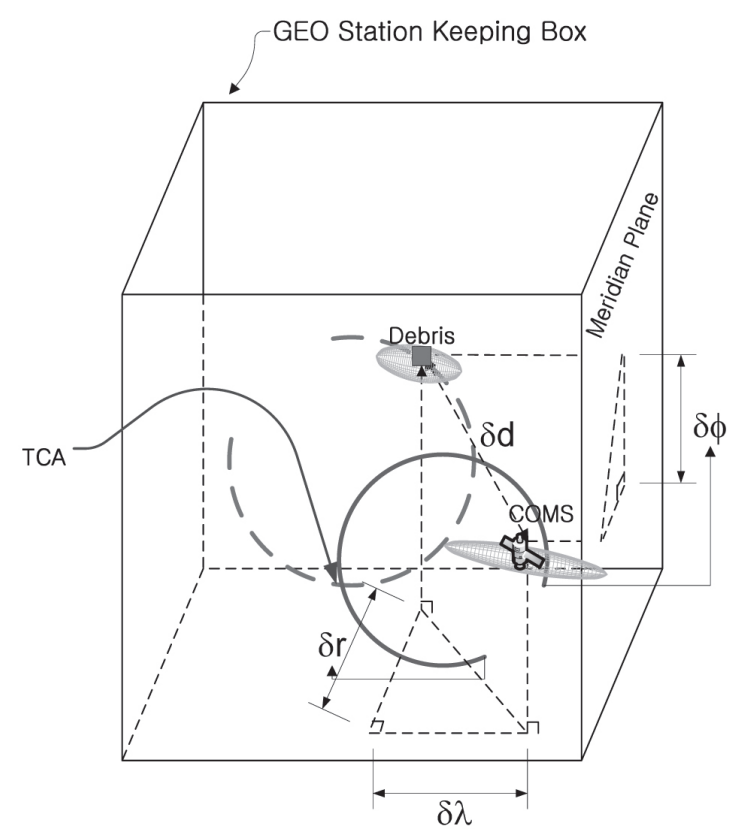

Fig. 2. Stationkeeping for GEO Satellites 
of the elements from previous generations in eachof the iterations. They are subjected to five basic operations to produce better results. Creation is the procedure of randomlygenerating a population. Reproduction is the process where each string is copied by considering the values of the adaptive penalty function. Crossover is the process where the combination of two chromosomes generates a new descendant. Mutation is the process where there is an occasional random modification for the value of one element of a chain. Epidemic is the process where a part of the population is exterminated with the consequent entrance of new individuals in the population[16].

Reproduction is assigned to those elements that have the highest value of the quantity chosen to be the measurement of fitness for an individual, and thus has a higher probability of contributing to the next generation, creating at least one descendant. The higher the values of this objective function, the higher are the chances that an individual will survive in the environment and reproduce its genetic material to the next generation. The procedure starts with a random population of up to several individuals. The initial population is generated randomly, taking into account distance, time, angle, acceleration, velocity, etc., according to the constraints of each variable[17].

\subsection{Collision Avoidance Maneuver Planning}

Usually, sun-synchronous satellites in LEO should be maintained within a ground track boundary for imaging missions, and GEO satellites for imaging and communication missions should be maintained in their own stationkeeping box. Therefore, planning for an avoidance maneuver should take into account the stationkeeping box or ground track boundary to obtain the maneuver start time and delta-V. Once a collision risk event is estimated, various avoidance maneuver plans should be generated. An acceptable avoidance maneuver plan must produce an orbit change that reduces the collision risk while still meeting positioning constraints to retain the stationkeeping box.

If a potential collision risk is greater than the maximum threshold, the possible collision events are listed and the operator is warned of the possible collision objects, time of closest approach, collision probability, range distance, etc. Then, a precise orbit is determined by using all antennas tracking data or the global position systemnavigation solution from our site as well as ephemeris from worldwide collaborating observation sites. An analytic collision prediction processor is used to determine which objects or debris might collide with the satellite by numerically integrating the equations of motion for each object. Then, the position of each object can be compared to that of every other object at each time step over the time span of interest. In this study, a minimum distance or collision probability for collision risk was evaluated. The collision probability was obtained from STK/AdvCAT which adopts the Alfano formula. The next step was to determine the maneuver start time and delta- $\mathrm{V}$ generated by a genetic algorithm with a boundary.

\section{Numerical Simulation}

\subsection{Simulation Setup}

The variables are delta- $\mathrm{V}$ and maneuver start time for one impulsive maneuver, where the boundary should be less than $\pm 1 \mathrm{~m} / \mathrm{s}$ for delta-V. The maneuver starting time before the time of closest approach should be less than $24 \mathrm{~h}$ in the case of the GEO satellite and $12 \mathrm{~h}$ for the LEO satellite. Table 1 and Table 2 list the initial state and epoch time of the GEO and the LEO satellite and debris for the avoidance maneuver simulation. Table 3 presents collision data at the time of closest approach.

Fig. 3 shows a maneuver planning flowchart. The STK/ Astrogator propagates with delta-V at the maneuver start time generated in the genetic algorithm and is integrated with an accurate perturbation model because the linearized perturbed model is not adequate for obtaining an accurate delta-V and time to ensure object avoidance. STK advCAT reports the range distance or collision probability between

Table 1. Initial State of the GEO satellite

\begin{tabular}{|c|c|c|}
\hline & GEO Sat & Debris \\
\hline Semi-major & 42165.7 & 42163.5 \\
\hline Eccentricity & $1 \mathrm{e}-005$ & $1 \mathrm{e}-005$ \\
\hline Inclination & 0.001 & 0.0001 \\
\hline Arg. of Perigee & 45 & 90 \\
\hline RAAN & 270 & 45 \\
\hline True Anomaly & 243.2 & 63 \\
\hline \multicolumn{2}{|c|}{ Epoch: June 1, 2013 12:00:00.000 } \\
\hline
\end{tabular}

Table 2. Initial State of the LEO satellite

\begin{tabular}{|c|c|c|}
\hline & LEO Sat & Debris \\
\hline Semi-major & 6937.66 & 7389.38 \\
\hline Eccentricity & 0.0012 & 0.07110 \\
\hline Inclination & 97.59 & 33.4791 \\
\hline Arg. of Perigee & 67.2353 & 295.958 \\
\hline RAAN & 339.484 & 6.73426 \\
\hline True Anomaly & 292.685 & 68.8462 \\
\hline \multicolumn{2}{|c|}{ Epoch: June 1, 2010 00:00:00.000 } \\
\hline
\end{tabular}


Table 3. Collision Data at Time of Closest Approach

\begin{tabular}{|c|c|c|}
\hline & GEO Case & LEO Case \\
\hline Estimated Time of Closest Approach & June 8, 2013 13:26:36.810 & June 2, 201012:40:00.338 \\
\hline Max. Collision Probability & $1.9429 \mathrm{e}-003$ & $2.6961 \mathrm{e}-002$ \\
\hline Radial $(\mathrm{km})$ & -2.172701 & -0.071910 \\
\hline Intrack $(\mathrm{km})$ & 0.643692 & -0.111136 \\
\hline Cross-track $(\mathrm{km})$ & 0.448201 & 0.065685 \\
\hline Distance $(\mathrm{km})$ & 2.309947 & 0.147773 \\
\hline
\end{tabular}

two conjugated objects or debris.

Table 4 lists the parameter values for setting the genetic algorithm. The fitness of each individual is represented by the total impulse velocity required to perform the orbital transfer. The population size is five $[18,19]$. The fitness function can be written as

$$
\mathrm{F}=\mathrm{C}_{1} \Delta \mathrm{f} / \mathrm{f}_{\text {nef }}+\mathrm{C}_{2} \Delta \mathrm{L}+\mathrm{C}_{3} \Delta \mathrm{P}_{\mathrm{c}}
$$

Inequality constraints for a LEO satellite are

$$
\begin{aligned}
& \mathrm{f}_{\text {nef }} \leq 0.3 \mathrm{~kg} \\
& -2 \mathrm{~km} \leq \Delta \mathrm{E} \leq+2 \mathrm{~km} \\
& 10^{-4} \leq \mathrm{P}_{\mathrm{c}} \leq 10^{-6}
\end{aligned}
$$

Inequality constraints for a GEO satellite are

$$
\begin{aligned}
& \mathrm{f}_{\text {nef }} \leq 0.3 \mathrm{~kg} \\
& -0.05^{\circ} \leq \Delta \lambda \leq 0.05^{\circ} \\
& -0.05^{\circ} \leq \Delta \phi \leq 0.05^{\circ} \\
& 10^{-4} \leq \mathrm{P}_{\mathrm{c}} \leq 10^{-6}
\end{aligned}
$$

where $\Delta \mathrm{f}$ denotes consumed fuel mass and $\mathrm{f}_{\text {nef }}$ is allowable fuel mass after the maneuver; $\Delta \mathrm{L}$ denotes the amount of deviation from a stationkeeping box for the GEO satellite

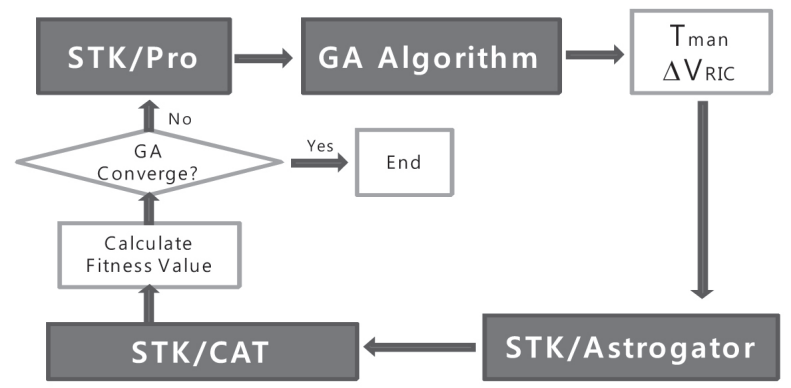

Fig. 3. Flowchart for Maneuver Planning or the deviation of a ground track boundary for the LEO satellite; $\mathrm{P}_{\mathrm{c}}$ is the logarithm value for the deviation of collision probability from the desired collision probability $\left(10^{-5}\right)$ between our satellite and objects; $C_{1}, C_{2}$ and $C_{3}$ are weighting factors, which are set to 100,10 , and 300 for the GEO satellite case and 100, 500, and 500 for the LEO satellite. However, if consumed fuel mass is less than the allowable fuel mass, $C_{1}$ is set to 0 ; if the satellite is not out of bound from its stationkeeping box or boundary after the maneuver, $C_{2}$ is set to 0 . Moreover, if $P_{C}$ has a value between $10^{-4}$ and $10^{-6}, C_{3}$ is set to 1 . In the above figures, $\Delta \mathrm{E}$ is the deviation in the ground track boundary for a LEO satellite; and $\Delta \lambda$ and $\Delta \phi$ represent the longitude and inclination angle from the stationkeeping box center, respectively. The first inequality constraints avoid collision risk, and the remaining ones limit

\begin{tabular}{|c|c|c|}
\hline & Parameter & Value \\
\hline \multirow{4}{*}{ Population } & Pop. Type & Double Vector \\
\hline & Pop. Size & 5 \\
\hline & Initialization & $\begin{array}{c}\text { Random with } \\
\text { Uniform }\end{array}$ \\
\hline & Creation Function & Uniform \\
\hline Selection & Function & Stochastic Uniform \\
\hline \multirow[b]{2}{*}{ Reproduction } & Elite Count & 2 \\
\hline & $\begin{array}{c}\text { Crossover } \\
\text { Fraction }\end{array}$ & 0.8 \\
\hline Crossover & Function & Scattered \\
\hline \multirow{3}{*}{ Mutation } & Function & Gaussian \\
\hline & Scale & 1 \\
\hline & Shrink & 1 \\
\hline $\begin{array}{l}\text { Stopping } \\
\text { Condition }\end{array}$ & $\begin{array}{l}\text { Maximum } \\
\text { Generation }\end{array}$ & 200 \\
\hline
\end{tabular}
stationkeeping box violation.

Increasing the population size enables the genetic algorithm to search for more points and thereby obtain a better result. However, the larger the population size, the longer the computation time for each generation. The elite count is the number of individuals with the best fitness values

Table 4. Parameter Setup for the Genetic Algorithm 
in the current generation that are guaranteed to survive in the next generation. These individuals are called elite children. The elite count is set to two. The crossover fraction is the fraction of individuals in the next generation, other than elite children, that are created by crossover. The rest are generated by mutation. The crossover fraction is set to 0.8 . Individuals with the lowest values reproduce too rapidly, taking over the population pool too quickly and preventing the genetic algorithm from searching other areas of the solution space. On the other hand, if the values vary only a little, all individuals have approximately the same chance of reproduction and the search will progress very slowly. The mutation scale parameter determines the variance in the first generation. The mutation shrink parameter controls how the variance shrinks as generations pass. If the shrink parameter is 0 , the variance is constant. If the shrink parameter is 1 , the variance shrinks linearly to 0 until the last generation is reached.

\subsection{Simulation Result of Avoidance Maneuver for GEO Satellite}

As shown in Fig. 4, space debris approaches from the West toward the GEO satellite operating normally in its stationkeeping box. If the satellite announces a possible collision by conjunction analysis software, the genetic algorithm attempts to find an optimal solution with constraints. The Genetic Algorithm Tool displays a plot of the best and mean values of the fitness function in each generation. When the algorithm stops, the plot appears as shown in Figs. 5 and 6. Points at the bottom of the plot denote the best fitness values, while points above them denote the averages of the fitness values in each generation. The plot also displays the best and mean values in the current generation at the top. Figs. 5 and 6 respectively display simulation results for East-West and North-South thrust used for the avoidance maneuver. Fig. 5 is converged with the best fit value of 2.6707 and the mean fit value of 2.6812 . Fig. 6 is converged with the best fit value of 2.7126 and the mean fit value of 2.713. Before the avoidance maneuver, the predicted range distance reached approximately $2.3 \mathrm{~km}$ and the collision probability was $1.94 \mathrm{e}-3$. It performed an orbital maneuver to steer its target satellite to prohibit any possible collision and interference from an uncontrolled object. After an avoidance maneuver was executed, the range distance increased to $4.72 \mathrm{~km}$ and subsequently drove the collision probability to $2.37 \mathrm{e}-5$ by using the NS thruster on June 7, 2013, 21:08:12 UTC before $15 \mathrm{~h} 43 \mathrm{~min}$ from the time of closest approach and a $6.58 \mathrm{~km}$ range distance. In the case

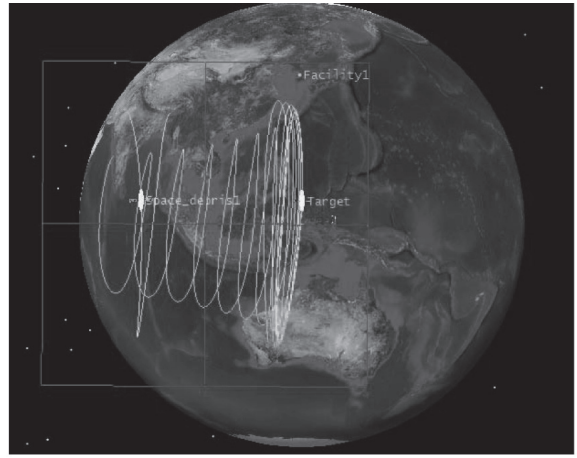

Fig. 4. 3D Graphics for GEO Satellite (EW Maneuver)

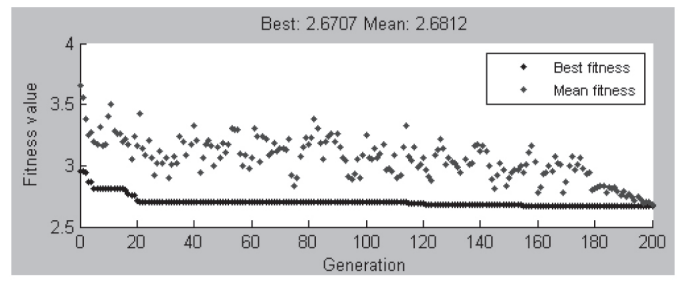

Fig. 5. Fitness Value for GEO Satellite (EW Maneuver)

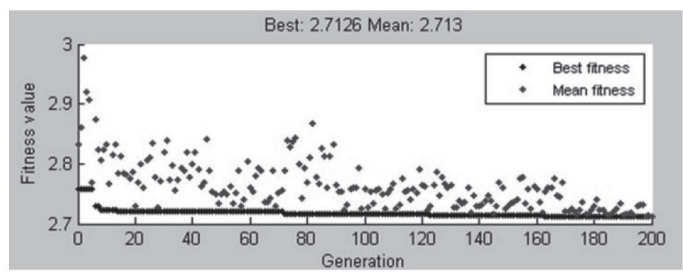

Fig. 6. Fitness Value for GEO Satellite (NS Maneuver)

Table 5. Avoidance Maneuver Case Study with Genetic Algorithm (GEO Satellite)

\begin{tabular}{|c|c|c|c|c|c|}
\hline & $\begin{array}{c}\Delta \mathrm{V} \\
(\mathrm{m} / \mathrm{s})\end{array}$ & Tman & $\begin{array}{c}\text { Min. } \\
\text { Range } \\
(\mathrm{km})\end{array}$ & $\begin{array}{c}\text { Collision } \\
\text { Prob. }\end{array}$ & $\begin{array}{c}\text { Used } \\
\text { Fuel } \\
(\mathrm{kg})\end{array}$ \\
\hline $\mathrm{I}(\mathrm{EW})$ & 0.0142 & $\begin{array}{c}\text { June 7, 2013 } \\
21: 08: 12 \\
(15: 43)\end{array}$ & 6.56 & $8.11 \mathrm{e}-5$ & 0.006 \\
\hline C(NS) & -0.6571 & $\begin{array}{c}\text { June 8,2013 } \\
09: 10: 48 \\
(3: 41)\end{array}$ & 4.72 & $2.37 \mathrm{e}-5$ & 0.2791 \\
\hline
\end{tabular}

Table 6. Avoidance Maneuver with Genetic Algorithm (Intrack Ma neuver)

\begin{tabular}{|c|c|c|c|c|c|}
\hline & $\begin{array}{c}\Delta \mathrm{V} \\
(\mathrm{m} / \mathrm{s})\end{array}$ & Tman & $\begin{array}{c}\text { Min. } \\
\text { Range } \\
(\mathrm{km})\end{array}$ & $\begin{array}{c}\text { Collision } \\
\text { Prob. }\end{array}$ & $\begin{array}{c}\text { Used } \\
\text { Fuel } \\
(\mathrm{kg})\end{array}$ \\
\hline Intrack & -0.0342 & $\begin{array}{c}\text { June 2, 2010 } \\
06: 00: 16 \\
(06: 40)\end{array}$ & $2.105 \mathrm{~km}$ & $1.328 \mathrm{e}-4$ & 0.015 \\
\hline
\end{tabular}




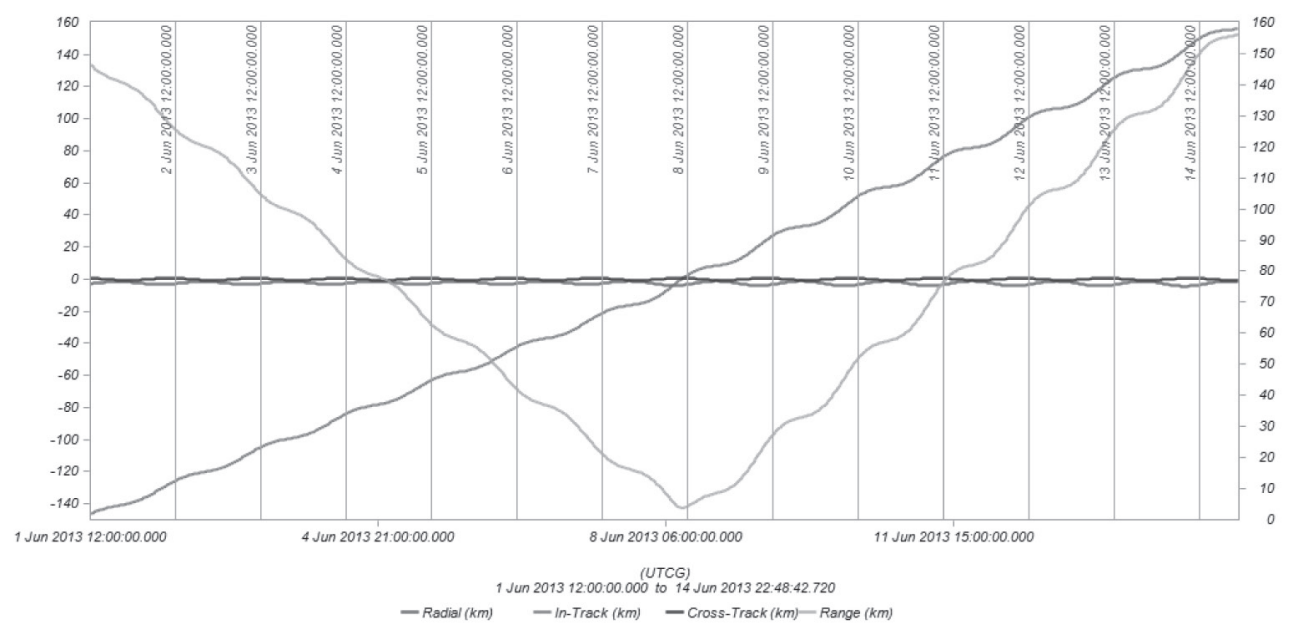

Fig. 7. Radial, Intrack, Cross-track, and Range Distances after the Collision Maneuver between Two Objects (EW Maneuver)

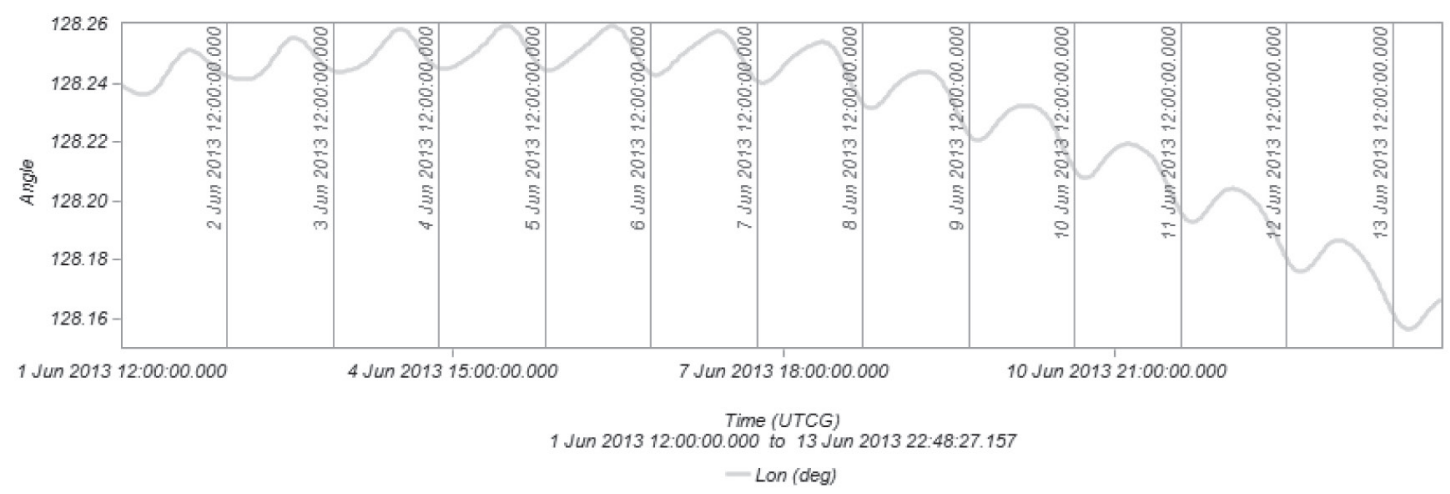

Fig. 8. Longitude Change after the Collision Maneuver for GEO Satellite (EW Maneuver)

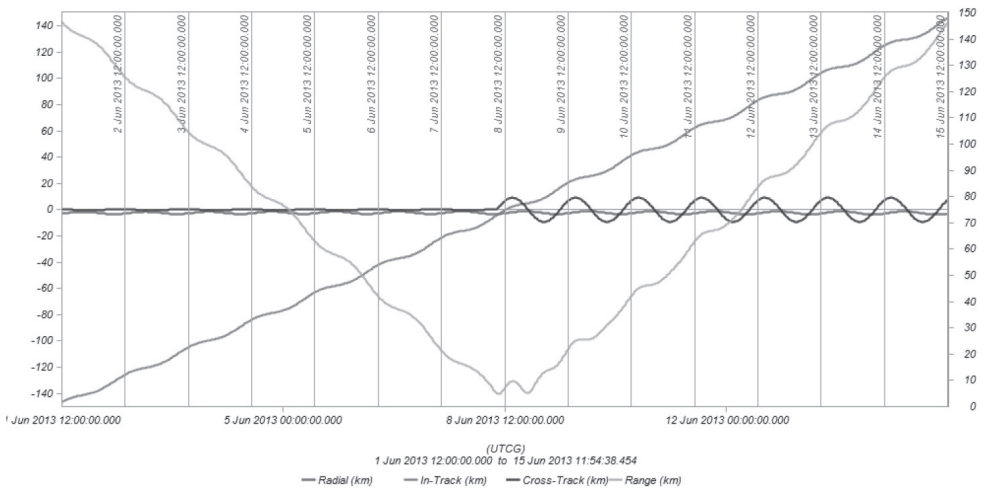

Fig. 9. Radial, Intrack, Cross-track.and Range Distances after the Collision Maneuver between Two Objects (NS Maneuver)

of East-West maneuver, the result was $8.11 \mathrm{e}-5$ of collision probability on June 8, 2013, 09:10:48 before $3 \mathrm{~h} 41 \mathrm{~min}$ from the time of closest approach.

Figs. 7 and 8 illustrate plots of a simulation result after the collision maneuver using the East-West maneuver for the GEO satellite. Fig. 8 shows the radial, intrack, cross-track, and range distances after the collision maneuver. The left side of the Y axis shows the radial, intrack, and cross-track distances between two objects, and the right side of the $\mathrm{Y}$ axis shows the range or miss distance between two objects. Fig. 8 presents longitude change after the collision maneuver. It shows that the longitude is kept at $128.2^{\circ} \mathrm{E}$ within the $\pm 0.05^{\circ}$ E/W stationkeeping box. Figs. 9 and 10 show the results of the collision maneuver using the North-South maneuver for the GEO satellite. Fig. 9 shows the radial, intrack, cross-track, and range distances after the collision maneuver. The left side of 


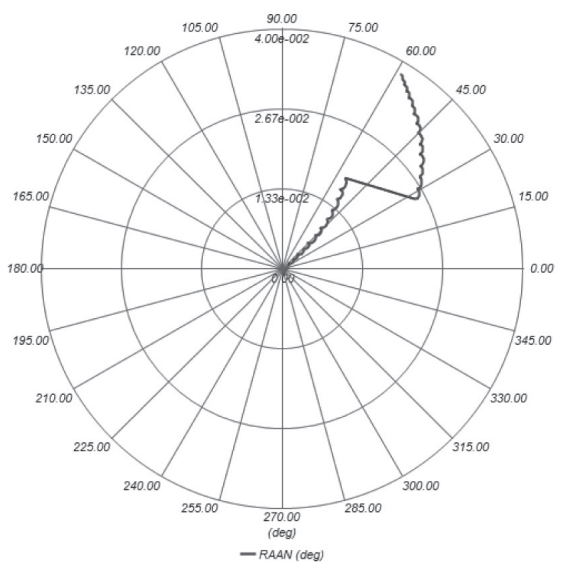

Fig. 10. Inclination Vector Change for GEO Satellite (NS Maneuver)

the $\mathrm{Y}$ axis shows the radial, intrack, and cross-track distances between two objects and the right side of the $\mathrm{Y}$ axis shows the miss distance between two objects. Fig. 10 shows the change in the inclination vector after the collision maneuver. It reveals that satellite longitude is retained within the $\pm 0.05^{\circ}$ $\mathrm{N} / \mathrm{S}$ stationkeeping box.After an avoidance maneuver, the range distance increased to $6.56 \mathrm{~km}$ for the EW maneuver and $4.72 \mathrm{~km}$ for the NS maneuver and subsequently drove the collision probability to 8.11e-5for the EW maneuver and 2.37e-5for the NS maneuver as shown in Table 5.

\subsection{Simulation Result of Avoidance Maneuver for LEO satellite}

The $I_{2}$ perturbation model includes the point mass effect as well as the dominant effect of asymmetry in the gravitational field. Therefore, this $I_{2}$ model is not sufficient, as shown in equations (1)-(4), to represent real perturbation, and a highprecision orbit propagator (HPOP) model is used in the STK to propagate and evaluate the ground track maintenance. The HPOP uses numerical integration of the differential equations of motion to generate an ephemeris. Several force

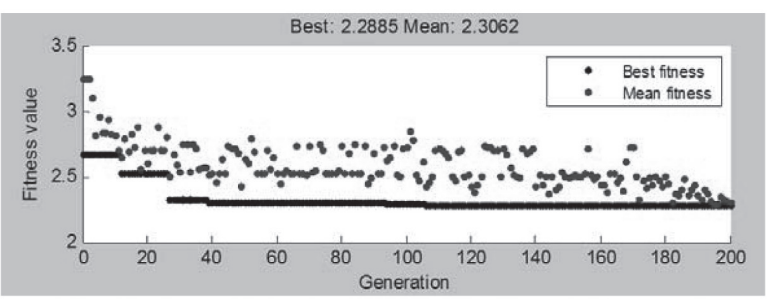

Fig. 12. Fitness Value for LEO Satellite (Intrack Maneuver)

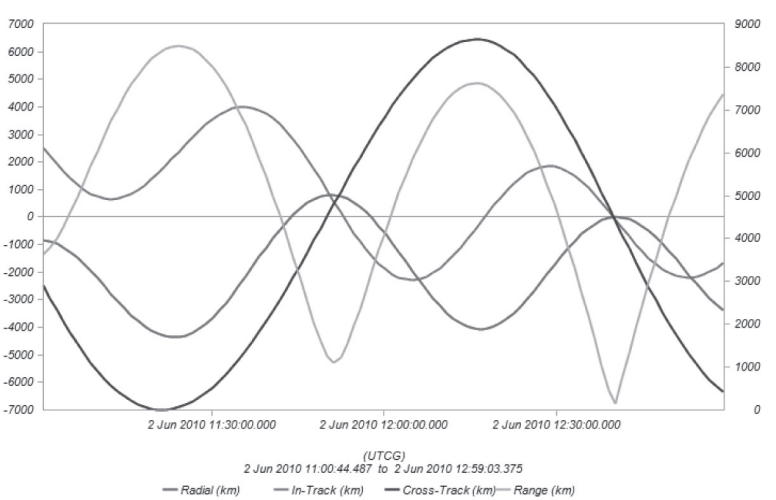

Fig. 13. Radial, Intrack, Cross-track, and Range Distances after the Collision Maneuver between Two Objects (Intrack Maneuver)

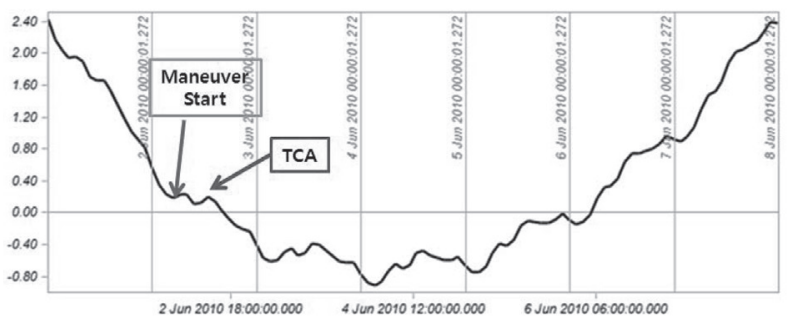

Fig. 14. Ground Track Error for LEO Satellite (Intrack Maneuver)

modeling effects can be included in the analysis, including a full gravitational field model (based on spherical harmonics), third-body gravity, atmospheric drag, and solar radiation pressure.In this study, the reference longitude is assumed to be $90.22^{\circ} \mathrm{E}$ for equator crossing points based on the camera's field of view, and the revisiting time from the initial ground track is set to 421 repeat counts.

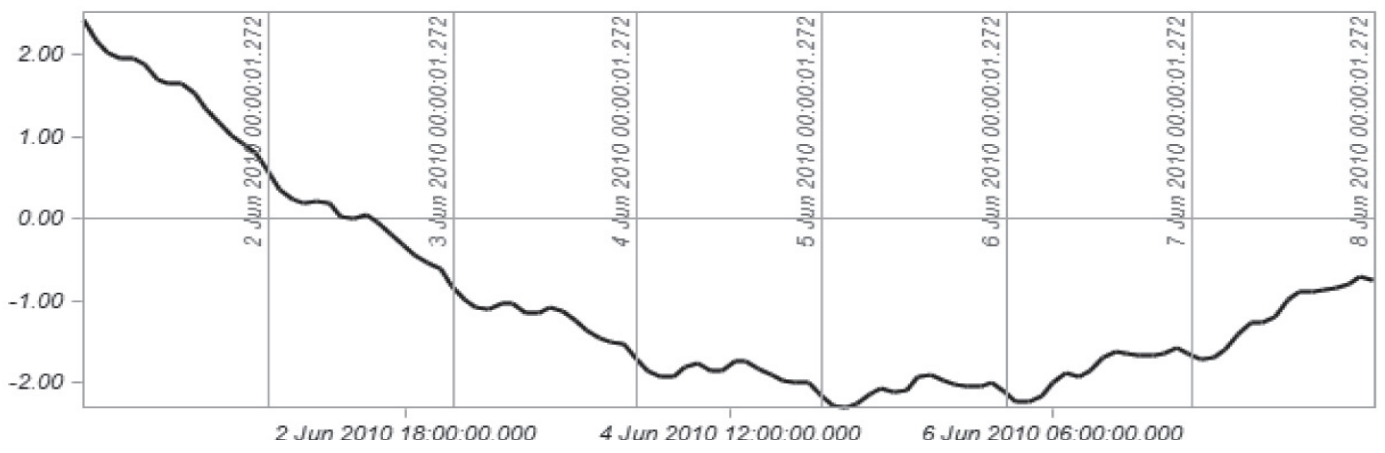

Fig. 11. Ground Track Error ofLEOSatellite(Before Maneuver) 
Fig. 11 shows ground track error before the collision maneuver. It indicates that the satellite's ground track is retained within the $\pm 2 \mathrm{~km}$ ground track boundary. If the satellite announces a possible collision by conjunction analysis software, the genetic algorithm attempts to find an optimal solution with constraints. Figs. 12-14 are plots of the simulation result after the collision maneuver using the intrack maneuver for the LEO satellite. Fig. 12 shows the best and mean values of the fitness function at the end of a generation. The plot is generated when the algorithm finds a solution, with the points at the bottom of the plot indicating the best fitness values and points above them indicating the averages of the fitness values in each generation. The plot also displays the best and mean values in the current generation at the top. This figure shows that after 200 generations, a best fit of 2.2479 and mean fit of 2.248 are obtained.

On June 2, the predicted range distance reached approximately $0.128 \mathrm{~km}$ and the collision probability was 0.0358. After an avoidance maneuver, the range distance increased to $2.105 \mathrm{~km}$ and subsequently drove the collision probability to $1.328 \mathrm{e}-4$ by using the intrack direction thruster at 06:40 from the time of closest approach, as shown in Table 6. Fig. 13 provides the radial, intrack, cross-track, and range distances after the collision maneuver. The left side of the $\mathrm{Y}$ axis shows the radial, intrack, and cross-track distances between two objects and the right side of the $\mathrm{Y}$ axis shows the miss distance between two objects. Fig. 14 shows ground track error after the collision maneuver. Furthermore, it shows that the ground track is retained within the $\pm 2 \mathrm{~km}$ ground track boundary.

\section{Conclusions}

In this study, a genetic algorithm was used to obtain maneuver start time and delta-V so as to reduce the probability of collision between approaching uncontrolled objects or debris and a LEO satellite and GEO satellite maintained in a stationkeeping box, while considering position uncertainty. The GEO satellite was stationed at $128.2^{\circ} \mathrm{E}$ within a $\pm 0.05^{\circ} \mathrm{E} / \mathrm{W}$ and N/S stationkeeping box, and the LEO satellite was maintained within $\pm 2.0 \mathrm{~km}$ ground track boundary having $312.4^{\circ} \mathrm{E}$ for equator crossing and 409 revolutions for repeat counts. An optimal maneuver solution was found to minimize fuel consumption and collision probability while considering position uncertainty. In the case of the GEO satellite, the predicted range distance reached approximately $2.3 \mathrm{~km}$ and the collision probability was $1.94 \mathrm{e}-3$. After the avoidance maneuver, the range distance increased to $4.72 \mathrm{~km}$ and subsequently drove the collision probability down to $2.37 \mathrm{e}^{-5}$. In the case of the LEO satellite, the predicted range distance reached approximately $0.23 \mathrm{~km}$ and the collision probability was 0.01 . After the avoidance maneuver, the range distance increased to 2.91 $\mathrm{km}$ and subsequently drove the collision probability down to $6.93 \mathrm{e}^{-5}$. The genetic algorithm showed good performance for avoidance maneuvers regarding LEO and GEO satellites. Grid computing technology and a graphics-processing unit will be adopted in the near future to improve computational efficiency. In addition, the structure of the genetic algorithm will be optimized to reduce computation time.

\section{Acknowledgements}

The research was supported by "Electro-optic Space Surveillance System" of the National Agenda Project funded by the Korea Research Council of Fundamental Science \& Technology. We would like to thank KRCFS and KARI for their support.

\section{References}

[1] Pimnoo, A., "The Satellite Collision Avoidance Strategy for THEOS Satellite", World Academy of Science, Engineering and Technology, 2011, pp.122-128.

[2] AGI, “Space Situational Awareness", STK Inview, 2008.

[3] NASA, "NASA and DARPA Sponsor International Debris Removal Conference", Orbital Debris Quarterly News, Vol.14, Issue 1, 2010.pp.1-2.

[4] X. Pena, C.W. Brown, "Operational Collision Risk Assessment of CALIPSO and LANDSAT-5 Crossings", SpaceOps 2012 Conference.

[5] D.P. Mckinley, "Maneuver Planning for Conjunction Risk Mitigation with Ground-track Control Requirements". NTRS, 2008.

[6] Kim, E. H., and Kim, E. D., "A Study on the Collision Avoidance Maneuver Optimization with Multiple Space Debris", JASS, Vol. 29, No. 1, 2012, pp. 11-21.

[7] Kim, H. D.,Kim, E. H., Kim, B. Y., Kim, H. Y.,andKim, H. J., "Analysis of Collision Probability of COMS-1 Satellite according to the Position Uncertainty", Proceeding of the 2011 KSAS Spring Conference, 2011.

[8] Kim, E. H., Kim, H. D., Kim, E. G., and Kim, H. J., "Analysis of Collision Avoidance Maneuver Frequency for the KOMPSAT-2 and KOMPSAT-5", KSAS, Vol.39, No.11, 2011,pp. 1033-1041.

[9] H.D. Kim, E.H. Kim, W.S. Uhm, E.K. Kim, H.J. Kim, "Conceptual Design of a Space Debris Collision Risk 
Management System", Proceeding of the 2011 KSAS Fall Conference, 2011.

[10] Duncan,M.,andWysack,J.,“Operational Collision Risk Management - Evaluating and Mitigating High-Risk Conjunction Events", AMOS Conference, 2011.

[11] Global Optimization Toolbox User's Guide,TheMathWorks Inc., 2010.

[12] Spacecraft Attitude and Orbit Control, Princeton Satellite System, 2009.

[13] Soop, E. M.,Hand Book of Geostationary Orbit, Kluwer Academic Publisher.

[14] S.C. Lee, T.S. No, "Optimal Separation Strategy for the Collocation of GEO Spacecrafts”, KSAS Paper, 1999. pp. 37-45.

[15] S.C. Lee, B.Y. Kim, B.K. Park, "A Study on the East-West
Station Keeping Box of Koreasat2", KSAS Paper, 2003, pp. 7581.

[16] Genetic Algorithm Toolbox User's Guide, The Math Works Inc., 2010.

[17] dos Santos, D.P.S., and Prado,A.F.B.A.,"Minimum Fuel Multi-Impulsive Orbital Maneuvers Using Genetic Algorithms", IAA-AAS-DyCoSS1-11-01.

[18] Kim, E. H., "A Study on the Optimization of Collision Avoidance Maneuver", Master'sThesis, University of Science \& Technology, 2012.

[19] Eckstein, M.C., Rajasingh, C. K.,andBlumer, P.,'Collocation Strategy and Collision Avoidance for the Geostationary Satellites at 19 Degrees West", CNES International Symposium on Space Dynamics, 1989. 